\title{
STRUCTURED SPATIAL DISCRETIZATION OF DYNAMICAL SYSTEMS
}

\author{
Marko Jokic $^{1}$, Andrej Jokic ${ }^{1}$, and Bruno Dogancic ${ }^{1}$ \\ ${ }^{1}$ University of Zagreb, Faculty of Mechanical Engineering and Naval Architecture \\ 10000 Zagreb, Ivana Lucica 5, Croatia \\ e-mail:mjokic@fsb.hr, ajokic@fsb.hr,bruno.dogancic@stud.fsb.hr
}

Keywords: Spatial Discretization, Structured Dynamical System, Uncertainty Modeling.

\begin{abstract}
This paper deals with spatial discretization of dynamical systems, such that the resulting discretized model is structured as a set of interconnected subsystems. We start by discretizing a dynamical system using an appropriate method (e.q. the finite element method) and discuss a general framework for partitioning a single discretized system into two interconnected systems. As it turns out, partitioning the discretized system into a set of interconnected systems appears to be a straightforward extension of the proposed procedure - we simply recursively apply the procedure. When doing so, we derive a general condition for preserving the interconnection structure, i.e. a necessary condition on the discretized system matrices such that the interconnection graph between the resulting subsystems matches the interconnection graph between the respective physical subdomains of the original system. This framework allows us to address a local discretization error (i.e. an error in a subsystem) influence on the overall system response. Furthermore, by considering a series of successively finer discretizations of an individual subsystem, we are able to construct a series of successively finer discretized system models with respective uncertainties.
\end{abstract}




\section{INTRODUCTION}

Spatial discretization of partial differential equations [1] describing a dynamical system has been one of the cornerstones of structural dynamics, vibration systems modeling, wave propagation studies, and so on for several decades. As the result, a plethora of techniques for modeling and simulation of dynamical systems, such as the finite element method [2, 3], have been extensively researched and established. Virtually all of the analysis techniques based on discretization rely on the assumption that a series of successively finer spatial discretizations of the system converge to the "accurate" mathematical model of the system. In this setting, choosing a spatial discretization which is accurate enough for a problem at hand usually boils down to a-posteriori analysis, i.e. addressing the error between a chosen discretization and an "accurate" model, usually a very fine discretization of the entire system.

On the other hand, robust control theory of dynamical systems adopts inherently different mathematical modeling paradigm - a dynamical system is approximated by a nominal system (e.q. linear time invariant system) and an uncertainty model [4]. The uncertainty model is typically a system with dynamic behaviour which confines a difference in behaviour between the nominal and the original system due to nonlinearities, neglected dynamics, discretization errors and so on. Roughly speaking, we are designing a simple model which captures all system behaviours, as opposed to structural dynamics approach where a (hopefully accurate) detailed and complex mathematical model is constructed. Structured control schemes, such as distributed and decentralized control [5], introduce additional mathematical modeling requirements. In structured control applications, a mathematical model of a system to be controlled comprises of a number of (possibly uncertain) interconnected dynamical systems, as shown in Figure 1 . Thus, the mathematical model exhibits a certain structure, usually represented by a graph graph vertices are subsystems and graph edges represent subsystems connections.

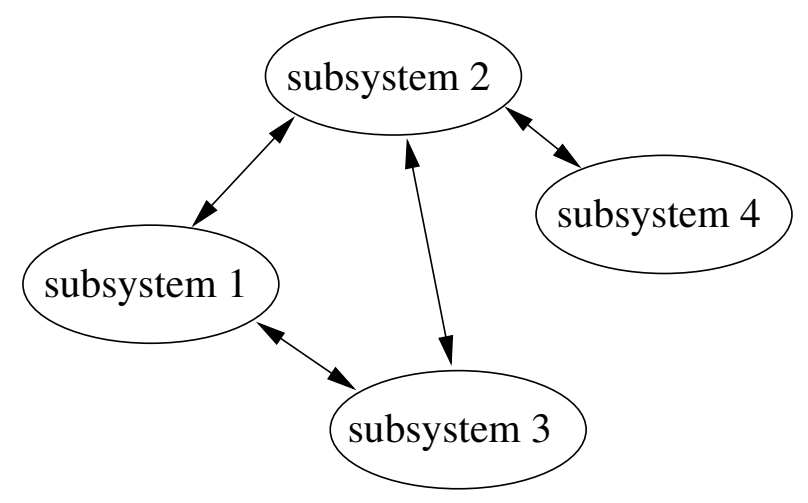

Figure 1: Structured representation of a dynamical system.

This paper addresses the following issue: How to construct a spatial discretization of a dynamical system, such that its discretized model has a structure corresponding to a given graph? We begin by providing a general framework for partitioning a discretized mechanical system into a series of interconnected mechanical systems. Then, we derive a necessary condition which, if met, allows us to use the partitioning procedure for constructing structured discretized model of a system. 


\section{PARTITIONING A SPATIALLY DISCRETIZED SYSTEM INTO A SET OF SUB- SYSTEMS}

Without loss of generality, we discuss a case of partitioning a single discretized mechanical system into two interconnected mechanical systems. As it turns out, partitioning a system into a series of interconnected systems may be viewed as a straightforward extension of the procedure.

Let us consider a mechanical system spatially discretized by means of the finite element method. In particular, we consider a linear time invariant (LTI) second order system

$$
\begin{gathered}
M \ddot{q}+C_{v} \dot{q}+K q=B_{d} d, \\
z=C x+D d,
\end{gathered}
$$

where $M, C_{v}$ and $K$ are mass, viscous damping and stiffness matrices, respectively, $B, C$ and

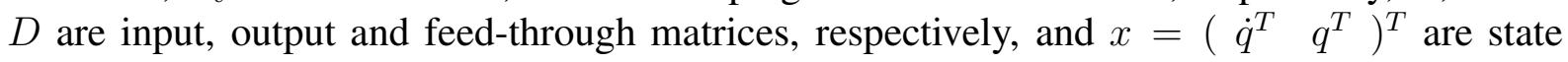
variables. The matrices $B, C$ and $D$ are input, output and feed-through matrices, and $d$ and $z$ are input and output vectors, respectively.

The system (1) may be rewritten as the equivalent first order descriptor system

$$
\begin{gathered}
E \ddot{x}=A x+B w, \\
z=C x+D w,
\end{gathered}
$$

with

$$
E=\left(\begin{array}{cc}
M & 0 \\
0 & F
\end{array}\right), \quad A=\left(\begin{array}{cc}
-C_{v} & -K \\
I & 0
\end{array}\right), \quad B=\left(\begin{array}{c}
B_{w} \\
0
\end{array}\right)
$$

where $F$ may be any nonsingular matrix (usually chosen to be the identity matrix $I$ ).

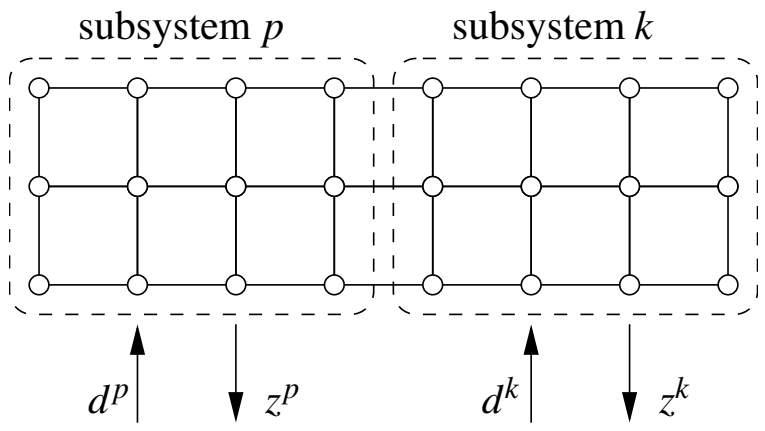

Figure 2: Partitioning the discretized system.

To partition the system (1) into two interconnected systems, we begin by identifying the boundary that separates the degrees of freedom (DOFs) of the two subsystems, which we denote by subsystem $p$ and subsystem $k$, as shown in Figure 2. Typically, when dealing with spatially discretized system by means of FEM, this is equivalent to identifying the nodes (with respective DOFs) belonging to either subsystem $p$ or subsystem $k$. More formally, we reorder the state variables $\tilde{x}$ such that $\tilde{x}=\left(\left(x^{p}\right)^{T}\left(x^{k}\right)^{T}\right)^{T}$, where $x^{p}$ and $x^{k}$ denote the state variables for the subsystem $p$ and subsystem $k$, respectively. In the similar fashion, we reorder the inputs $w$ and the outputs $z$ such that $\tilde{w}=\left(\left(w^{p}\right)^{T} \quad\left(w^{k}\right)^{T}\right)^{T}$ and $\tilde{z}=\left(\left(z^{p}\right)^{T}\left(z^{k}\right)^{T}\right)^{T}$, where $p$ and $k$ denote the variables for the subsystem $p$ and subsystem $k$, respectively.

Furthermore, we assume that a single component $d_{i}$ of the input vector $d=\left(\begin{array}{lllll}d_{1} & d_{2} & \cdots & d_{n}\end{array}\right)^{T}$ acts either on subsystem $p$ or the subsystem $k$, but not on the both subsystems. If this is not the case, i.e. if the signal $d_{i}$ acts on both subsystems, it can be split into two signals $\left(d^{p}\right)_{i}$ and 
$\left(d^{k}\right)_{i}$ acting on subsystems $p$ and $k$, respectively. Equivalently, if the output signal $z_{i}$ originates form both subsystems, it can be split into two output signals $\left(z^{p}\right)_{i}$ and $\left(z^{k}\right)_{i}$ originating from subsystems $p$ and $k$, respectively. We also assume that the feed-through matrix $D$ maps the inputs that act on a single subsystem only to the outputs that correspond to the same subsystem - if this is not the case, the matrix $D$ may be modified in the similar fashion as described above.

The above described procedure may be accomplished by constructing a permutation matrix $P^{p k}$ such that

$$
\tilde{x}=\left(\begin{array}{c}
x^{p} \\
x^{k}
\end{array}\right)=P^{p k} x, \quad \tilde{d}=\left(\begin{array}{c}
d^{p} \\
d^{k}
\end{array}\right)=P^{p k} d, \quad \tilde{z}=\left(\begin{array}{c}
z^{p} \\
z_{k}
\end{array}\right)=P^{p k} z .
$$

By multiplying the equation (2) by the matrix $P^{p k}$ from the left and taking into the account (4), we obtain

$$
\begin{gathered}
\tilde{E} \ddot{\tilde{x}}=\tilde{A} \tilde{x}+\tilde{B} \tilde{d}, \\
\tilde{z}=\tilde{C} \tilde{x}+\tilde{D} \tilde{d},
\end{gathered}
$$

where

$$
\begin{gathered}
\tilde{E}=P^{p k} E\left(P^{p k}\right)^{T}, \quad \tilde{A}=P^{p k} A\left(P^{p k}\right)^{T}, \quad \tilde{B}=P^{p k} B\left(P^{p k}\right)^{T}, \\
\tilde{C}=P^{p k} C\left(P^{p k}\right)^{T}, \quad \tilde{D}=P^{p k} D\left(P^{p k}\right)^{T},
\end{gathered}
$$

where

$$
\tilde{E}=\left(\begin{array}{cc}
E^{p p} & E^{p k} \\
E^{k p} & E^{k k}
\end{array}\right), \quad \tilde{A}=\left(\begin{array}{cc}
A^{p p} & A^{p k} \\
A^{k p} & A^{k k}
\end{array}\right) .
$$

Due to our previous assumptions (i.e. a single input acts only on the subsystem $p$ or the subsystem $k$, a single output originates only from the subsystem $p$ or the subsystem $k$ and the feed-through matrix maps the inputs that act on a single subsystem only to the outputs that correspond to the same subsystem), the matrices $\tilde{B}, \tilde{C}$ and $\tilde{D}$ are block-diagonal

$$
\tilde{B}=\left(\begin{array}{cc}
B^{p} & 0 \\
0 & B^{k}
\end{array}\right), \quad \tilde{C}=\left(\begin{array}{cc}
C^{p} & 0 \\
0 & C^{k}
\end{array}\right), \quad \tilde{D}=\left(\begin{array}{cc}
D^{p} & 0 \\
0 & D^{k}
\end{array}\right) .
$$

Consequently, we have two subsystems that are coupled through both matrices $\tilde{E}$ and $\tilde{A}$. The subsystem $p$ is defined by

$$
\begin{gathered}
E^{p p} \dot{x}^{p}+E^{p k} \dot{x}^{k}=A^{p p} x^{p}+A^{p k} x^{k}+B^{p} d^{p}, \\
z^{p}=C^{p} x^{p}+D^{p} d^{p},
\end{gathered}
$$

and the subsystem $k$ is defined by

$$
\begin{gathered}
E^{k p} \dot{x}^{p}+E^{k k} \dot{x}^{k}=A^{k p} x^{p}+A^{k k} x^{k}+B^{k} d^{k}, \\
z^{k}=C^{k} x^{k}+D^{k} d^{k} .
\end{gathered}
$$

We eliminate $\dot{x}^{k}$ from $(9)$ and $\dot{x}^{p}$ from $(10)$, and after some manipulation we finally obtain two interconnected subsystems. The subsystem $p$ is defined by

$$
\begin{gathered}
E^{p} \ddot{x}^{p}=A^{p} x^{p}+v^{p k}+B^{p} d^{p}, \\
z^{p}=C^{p} x^{p}+D^{p} d^{p}, \\
w^{p k}=C^{p k} x^{p}+D^{p k} d^{p},
\end{gathered}
$$




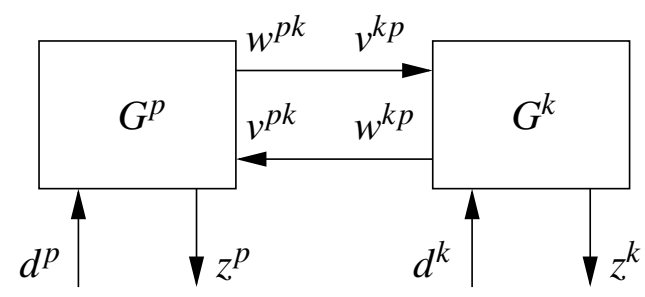

Figure 3: Interconnected systems.

where

$$
\begin{aligned}
E^{p}=E^{p p}-E^{p k}\left(E^{k k}\right)^{-1} E^{k p}, & A^{p}=A^{p p}-E^{p k}\left(E^{k k}\right)^{-1} A^{k p}, \\
C^{p k}=A^{k p}-E^{k p}\left(E^{p p}\right)^{-1} A^{p p}, & D^{p k}=-E^{k p}\left(E^{p p}\right)^{-1} B^{p} .
\end{aligned}
$$

In the similar fashion, the subsystem $k$ is defined by

$$
\begin{gathered}
E^{k} \ddot{x}^{k}=A^{k} x^{k}+v^{k p}+B^{k} d^{k}, \\
z^{k}=C^{k} x^{k}+D^{k} d^{k}, \\
w^{k p}=C^{k p} x^{k}+D^{k p} d^{k},
\end{gathered}
$$

where

$$
\begin{aligned}
E^{k} & =E^{k k}-E^{k p}\left(E^{p p}\right)^{-1} E^{p k}, & A^{k}=A^{k k}-E^{k p}\left(E^{p p}\right)^{-1} A^{p k}, \\
C^{k p} & =A^{p k}-E^{p k}\left(E^{k k}\right)^{-1} A^{k k}, & D^{k p}=-E^{p k}\left(E^{k k}\right)^{-1} B^{k} .
\end{aligned}
$$

By setting $v^{k p}=w^{p k}$ and $v^{p k}=w^{k p}$, the subsystems $p$ and $k$ are interconnected LTI systems with corresponding transfer functions $G^{p}$ and $G^{k}$, as shown in Figure 3 .

Note that the terms $E^{p k}$ and $E^{k p}$ in the above equations are due to the coupling terms in the inertia matrices of the subsystems $p$ and $k$. To be more specific, the terms $E^{p k}$ and $E^{k p}$ are due to the nondiagonal terms in the inertia matrices of the finite elements that contain DOFs from both subsystems $p$ and $k$. Consequently, if the inertia matrices of the finite elements that contain both $x^{p}$ and $x^{k}$ are lumped (i.e. diagonal), the terms $E^{p k}$ and $E^{k p}$ vanish and the interconnected subsystems are defined by

$$
\begin{gathered}
E^{p p} \dot{x}^{p}=A^{p p} x^{p}+v^{p k}+B^{p} d^{p}, \\
z^{p}=C^{p} x^{p}+D^{p} d^{p} \\
w^{p k}=A^{k p} d^{p}
\end{gathered}
$$

and

$$
\begin{gathered}
E^{k k} \dot{x}^{k}=A^{k k} x^{k}+v^{k p}+B^{k} d^{k}, \\
z^{k}=C^{k} x^{k}+D^{k} d^{k} \\
w^{k p}=A^{p k} d^{k} .
\end{gathered}
$$

Partitioning an arbitrary discretized system into a set of interconnected systems appears to be a straightforward extension of the above described procedure - we simply recursively apply the procedure to divide the resulting subsystems into a desired set of (new) subsystems. However, if we also require that the interconnection graph between the resulting subsystems matches the interconnection graph of the respective physical domains, the inertia matrices must meet a certain condition. We illustrate this in the following example. 


\section{EULER BEAM AS A STRUCTURED DISCRETIZED SYSTEM}

Consider an Euler beam subject to a series of concentrated loads, as shown in Figure 4 . If the distance between each two adjacent loads equals $l$, and the beam properties (flexural rigidity $E I$, cross section area $A$ and density $\rho$ ) are constant, we may view the beam as an assembly of four identical segments, which we denote by 1 through 4 . The remaining parts of the beam on the left and the right side are denoted by $L$ and $R$, respectively. By specifying the desired outputs (e.q. beam displacements at the positions of the loads) and after the discretization, as described in the previous sections, we end up with the LTI second order system (1) which represents the entire beam.

For the purpose of our discussion, we wish to partition the resulting LTI system into a set of subsystems, such that each subsystem represents individual beam segments $(L, R$ and 1 thorough 4). Furthermore, we also require that the interconnection graph for the resulting subsystems matches the interconnection graph for the actual beam segments, as shown in Figure 5. Without making any assumptions regarding the beam discretization, we proceed by recursively partitioning the LTI system that represents the entire beam.

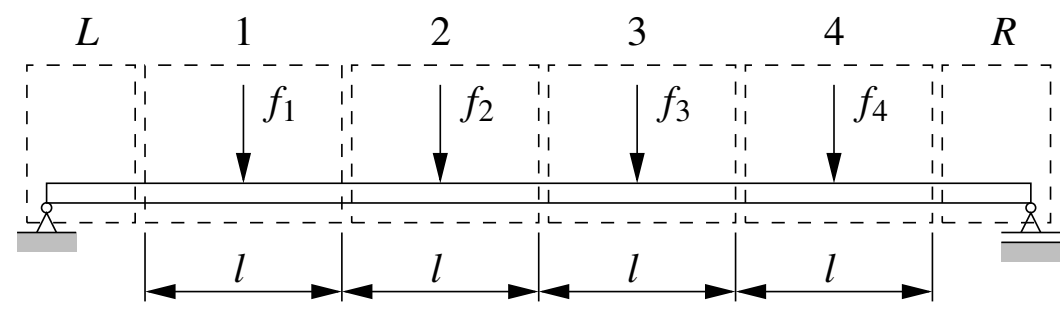

Figure 4: Euler beam as an assembly of segments.

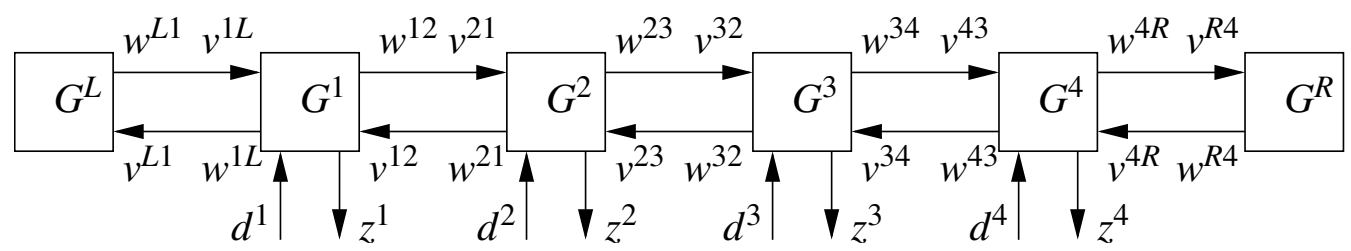

Figure 5: Euler beam as a series of interconnected systems.

First, we partition the system into the subsystem $G^{L}$ that represents the segment $L$, and the subsystem $G^{K}$ that represents the remaining segments (segments 1 through 4 and $R$ ). The resulting interconnected systems are shown in Figure 6, and the subsystems are defined as

- subsystem $G^{L}$ :

$$
\begin{gathered}
E^{L} \ddot{x}^{L}=A^{L} x^{L}+v^{L K}, \\
w^{L K}=C^{L K} x^{L},
\end{gathered}
$$

- subsystem $G^{K}$ :

$$
\begin{gathered}
E^{K} \ddot{x}^{K}=A^{K} x^{K}+v^{K L}+B^{K} d^{K}, \\
z^{K}=C^{K} x^{K}+D^{K} d^{K}, \\
w^{K L}=C^{K L} x^{K}+D^{K L} d^{K} .
\end{gathered}
$$

Now, let us take a closer look at the signal $v^{K L}=w^{L K}=C^{L K} x^{L}$. Observe that the matrix

$$
C^{L K}=A^{K L}-E^{K L}\left(E^{L L}\right)^{-1} A^{L L}
$$




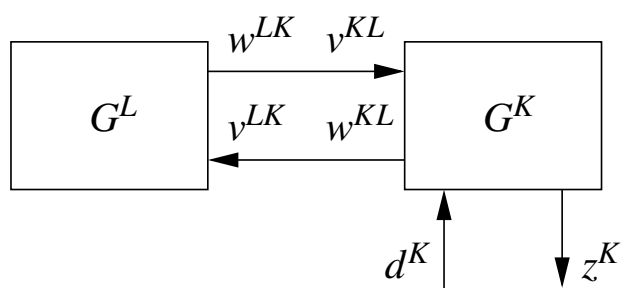

Figure 6: First partition of the beam.

contains the term $-E^{K L}\left(E^{L L}\right)^{-1} A^{L L}$, which has no special structure (i.e. it is full) due of the presence of $\left(E^{L L}\right)^{-1}$. The fact that $E^{L L}$ represents a part of the inertia matrix which is sparse and banded does not help here - its inverse is full matrix, as we illustrate on the Figure 7 , Consequently, the matrix $C^{L K}$ is full and all state variables $x^{L}$ are fed via $v^{K L}$ to all DOFs of the subsystem $K$.
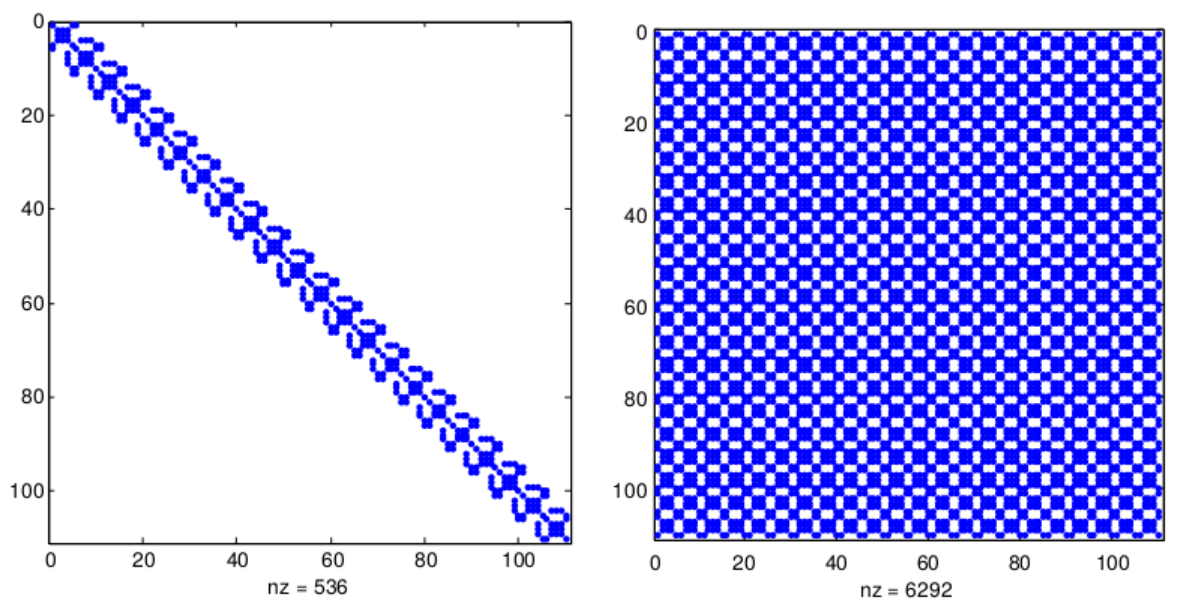

Figure 7: Nonzero entries of the inertia matrix (left) and its inverse (right).

Next, let us further partition the subsystem $G^{K}$ into a subsystem $G^{1}$ that represents the beam segment 1 and a subsystem $G^{M}$ that represents the beam segments 2 through 4 and $R$. Due to the fact that $x^{L}$ are fed to all DOFs of the subsystems $G^{1}$ and $G^{M}$, i.e. the subsystems that make up the subsystem $G^{K}$, we have the interconnection graph shown on Figure 8 . Further partitioning of the system would result in a set of subsystems where each subsystem is connected to all remaining subsystems.

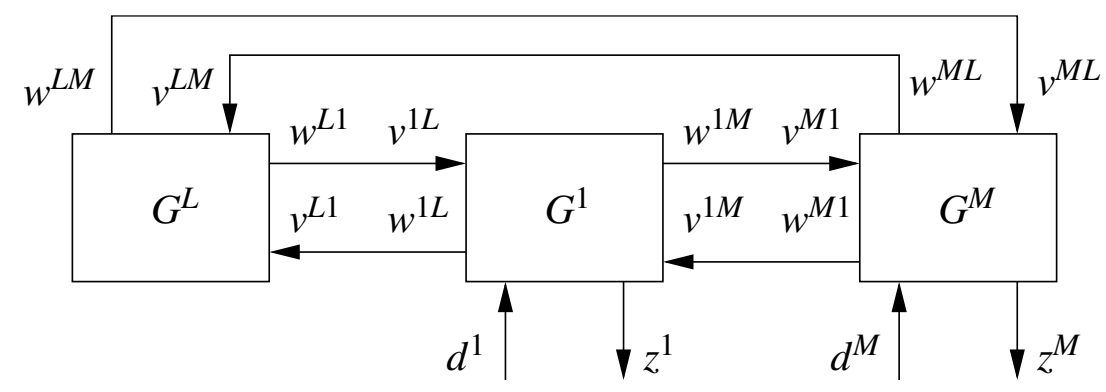

Figure 8: Second partition of the beam.

To remedy this, let us go back to the equation (19). Recall that when the inertia matrices of 
the finite elements that contain both $x^{L}$ and $x^{K}$ are lumped, the terms $E^{K L}$ vanishes and we have sparse and structured

$$
C^{L K}=A^{K L} .
$$

In this case, the state variables $x^{L}$ are fed via $v^{K L}$ only to the DOFs of the subsystem $K$ which belong to the elements that contain DOFs from both subsystems $L$ and $K$. In other words, only the states exchanged between the systems are the states of the DOFs at the boundaries between the systems. By taking this requirement into the account, recursive partitioning of the system results in a set of subsystems with the desired interconnection structure shown in Figure 5.

Thus, we conclude our discussion with a general condition for preserving the interconnection graph for a partitioned discretized system: an interconnection graph for a set of subsystems obtained by partitioning a discretized system of will have the same interconnection graph as the subdomains of the discretized system if the elements that represent the boundaries between the subsystems have lumped inertia matrices.

\section{CONCLUSIONS}

In the discussion above, we have presented a general framework for partitioning a linear time-invariant system system (e.q. a system obtained by means of the finite element method) into a set of interconnected systems. In doing so, we have derived a general condition for preserving the interconnection structure, i.e. a necessary condition on the discretized system matrices such that the interconnection graph between the resulting subsystems matches the interconnection graph between the respective physical subdomains. This framework allows us to address a local discretization error (i.e. an error in a subsystem) influence on the overall system response - for a possible approach, please refer to [6]. Furthermore, by considering a series of successively finer discretizations of an individual subsystem, we are able to construct a series of successively finer discretized system models with respective uncertainties.

\section{ACKNOWLEDGMENTS}

This work has been fully supported by Croatian Science Foundation under the project number 9354, Control of Spatially Distributed Systems.

\section{REFERENCES}

[1] C. Grossmann, H.-G. Roos, Numerical Treatment of Partial Differential Equations. Springer-Verlag, 2007.

[2] K.-J. Bathe, Finite Element Procedures. Prentice Hall, 1996.

[3] R.D. Cook, D.S. Malkus, M.E. Plesha, R.J. Witt, Concepts and Applications of Finite Element Analysis, 4th Edition. John Wiley \& Sons, 2002.

[4] G.E. Dullerud, F. Paganini, A Course in Robust Control Theory - A Convex Approach. Springer-Verlag, 2000.

[5] D. Siljak, Decentralized Control of Complex Systems. Academic Press, 1991.

[6] B.L. Jones, E.C. Kerrigan, When is the discretization of a spatially distributed system good enough for control?. Automatica, 46(9), 1462-1468, 2010. 